\title{
Some Hecke algebra products and corresponding random walks
}

\author{
Rosena R.X. Du • Richard P. Stanley
}

Received: 8 October 2008 / Accepted: 16 June 2009 / Published online: 7 July 2009

(C) Springer Science+Business Media, LLC 2009

\begin{abstract}
Let $\boldsymbol{i}=1+q+\cdots+q^{i-1}$. For certain sequences $\left(r_{1}, \ldots, r_{l}\right)$ of positive integers, we show that in the Hecke algebra $\mathcal{H}_{n}(q)$ of the symmetric group $\mathfrak{S}_{n}$, the product $\left(1+\boldsymbol{r}_{1} T_{r_{1}}\right) \cdots\left(1+\boldsymbol{r}_{l} T_{r_{l}}\right)$ has a simple explicit expansion in terms of the standard basis $\left\{T_{w}\right\}$. An interpretation is given in terms of random walks on $\mathfrak{S}_{n}$.
\end{abstract}

Keywords Hecke algebra $\cdot$ Tight sequence $\cdot$ Reduced decomposition $\cdot$ Random walk

\section{Main results}

Let $\mathfrak{S}_{n}$ denote the symmetric group of permutations of $\{1, \ldots, n\}$. For $1 \leq i \leq n-1$ let $s_{i}=(i, i+1) \in \mathfrak{S}_{n}$, the adjacent transposition interchanging $i$ and $i+1$ and leaving all the other elements fixed. For any $w \in \mathfrak{S}_{n}$ denote by $\ell(w)$ the length of $w$, i.e., the minimal $p$ such that $w$ can be written as

$$
w=s_{r_{1}} s_{r_{2}} \cdots s_{r_{p}}
$$

Rosena Du is partially supported by the National Science Foundation of China under Grant No. 10801053 and No. 10671074. Research carried out when she was a Visiting Scholar at M.I.T. during the 2007-2008 academic year. Richard Stanley's contribution is based upon work supported by the National Science Foundation under Grant No. DMS-0604423. Any opinions, findings and conclusions or recommendations expressed in this material are those of the author and do not necessarily reflect those of the National Science Foundation.

R.R.X. Du

Department of Mathematics, East China Normal University, Shanghai 200041, China e-mail: rxdu@math.ecnu.edu.cn

R.P. Stanley $(\bowtie)$

Department of Mathematics, M.I.T., Cambridge, MA 02139, USA

e-mail: rstan@math.mit.edu 
for certain $r_{1}, r_{2}, \ldots, r_{p}$; such a sequence $r=\left(r_{1}, \ldots, r_{p}\right)$ is called a reduced decomposition (or reduced word) provided $p=\ell(w)$.

The Hecke Algebra (or Iwahori-Hecke algebra) $\mathcal{H}_{n}(q)$ of the symmetric group $\mathfrak{S}_{n}$ (e.g., $\left.[5, \S 7.4]\right)$ is defined as follows: $\mathcal{H}_{n}(q)$ is an $\mathbb{R}$-algebra with identity 1 and generators $T_{1}, T_{2}, \ldots, T_{n-1}$ which satisfy relations

$$
\begin{aligned}
\left(T_{i}+1\right)\left(T_{i}-q\right) & =0, \\
T_{i} T_{j} & =T_{j} T_{i}, \quad|i-j| \geq 2, \\
T_{i} T_{i+1} T_{i} & =T_{i+1} T_{i} T_{i+1}, \quad 1 \leq i \leq n-2 .
\end{aligned}
$$

For any $w=s_{r_{1}} s_{r_{2}} \cdots s_{r_{p}} \in \mathfrak{S}_{n}$ for which $\left(r_{1}, r_{2}, \ldots, r_{p}\right)$ is reduced, define $T_{w}=$ $T_{r_{1}} T_{r_{2}} \cdots T_{r_{p}}$. A basic property of Hecke algebras is that $T_{w}$ does not depend on the choice of reduced decomposition of $w$, and for $1 \leq k \leq n-1, T_{w}$ satisfies

$$
T_{w} T_{k}= \begin{cases}T_{w s_{k}}, & \text { if } \ell\left(w s_{k}\right)=\ell(w)+1 \\ q T_{w s_{k}}+(q-1) T_{w}, & \text { if } \ell\left(w s_{k}\right)=\ell(w)-1\end{cases}
$$

Let $r=\left(r_{1}, r_{2}, \ldots, r_{l}\right)$ be any sequence of positive integers (not necessarily reduced). For convenience assume that $\max \left\{r_{1}, \ldots, r_{l}\right\}=n-1$. Set

$$
i=1+q+\cdots+q^{i-1} .
$$

For any $w \in \mathfrak{S}_{n}$, define $\alpha_{r}(w) \in \mathbb{Z}[q]$ by

$$
Q(r):=\left(1+\boldsymbol{r}_{1} T_{r_{1}}\right)\left(1+\boldsymbol{r}_{2} T_{r_{2}}\right) \cdots\left(1+\boldsymbol{r}_{l} T_{r_{l}}\right)=\sum_{w \in \mathfrak{S}_{n}} \alpha_{r}(w) T_{w} .
$$

We are primarily concerned with the polynomials $\alpha_{r}(w)$. In particular, for which $r$ 's will $\alpha_{r}(w)$ have "nice" values for all $w \in \mathfrak{S}_{n}$ ?

For each $w=w_{1} w_{2} \cdots w_{n} \in \mathfrak{S}_{n}$, we write $w \preceq r$ if $w=s_{c_{1}} \cdots s_{c_{k}}$ for some subsequence $c_{1}, \ldots, c_{k}$ of $r=\left(r_{1}, \ldots, r_{l}\right)$. This defines the Bruhat order on $\mathfrak{S}_{n}[5, \S 5.9]$. It follows from equation (2) that $\alpha_{r}(w)=0$ unless $w \preceq r$. Let $a_{r}(i)$ denote the number of $i$ 's in $r$, and let $\operatorname{inv}(w)=\left(\operatorname{inv}_{w}(1), \operatorname{inv}_{w}(2), \ldots, \operatorname{inv}_{w}(n-1)\right)$ denote the $i n$ version sequence of $w$, i.e., for any $1 \leq i \leq n-1, \operatorname{inv}_{w}(i)$ is the number of $j$ 's such that $w_{j}<w_{i}$ and $j>i$.

We recursively define a sequence $r=\left(r_{1}, \ldots, r_{l}\right)$ to be a tight sequence if it satisfies the following:

1. $r_{1}=1$;

2. If $r$ is a tight sequence, and $k$ is a positive integer, then $r^{\prime}=(r, k)$ (the concatenation of $r$ and $k$ ) is also a tight sequence if $a_{r}(k) \leq a_{r}(k-1)-1$, and equality holds when there exists a permutation $w$ satisfying $w \preceq r^{\prime}$ but $w \npreceq r$.

For example, there are 6 tight sequences of length 4: 1111, 1211, 1231, 1212, 1213,1234 . And any prefix of the sequences $(1,2,1,3,2,1, \ldots)$ or $(1,2, \ldots, n$, $1,2, \ldots, n-1, \ldots, 1,2,1)$ is a tight sequence. 
The main result of this paper is the following.

Theorem 1 Let $r$ be a tight sequence with $\max \{r\}=n-1$. Then for any $w \in \mathfrak{S}_{n}$ and $w \preceq r$, we have

$$
\alpha_{r}(w)=\prod_{i=2}^{n-1} i^{\max \left\{a_{r}(i-1)-1, \operatorname{inv}_{w}(i)\right\}}
$$

\section{Example 1}

(a) Define the standard tight sequence $\rho_{n}$ of degree $n$ by

$$
\rho_{n}=(1,2,1,3,2,1,4,3,2,1, \ldots, n-1, n-2, \ldots, 1) .
$$

It is easy to see that $\rho_{n}$ is not only a tight sequence but also a reduced decomposition of the element $w_{0}=n, n-1 \ldots, 1 \in \mathfrak{S}_{n}$. Theorem 1 becomes

$$
\alpha_{\rho_{n}}(w)=\mathbf{1}^{n-1} \mathbf{2}^{n-2} \cdots(\boldsymbol{n}-\mathbf{1}),
$$

independent of $w \in \mathfrak{S}_{n}$.

(b) Let $r=(1,2,1,1,3,1)$. Then we have

$$
\alpha_{r}(w)=\mathbf{2}^{3}, \quad \forall w \in\{1234,1324,2134,2314,3124,3214\}
$$

and

$$
\alpha_{r}(w)=\mathbf{2}^{3} \mathbf{3}, \quad \forall w \in\{1243,1342,2143,2341,3142,3241\} .
$$

Otherwise we have $\alpha_{r}(w)=0$.

Alexander Molev has pointed out (private communication dated September 1, 2008) that Theorem 1 in the case of the standard tight sequence is connected to the "fusion procedure" for the Hecke algebra, which goes back to Cherednik [1, 2].

\section{Proof of the main theorem}

For the proof of Theorem 1 we need the following lemma.

Lemma 1 Let $r=\left(r_{1}, \ldots, r_{l}\right) \in \mathbb{P}^{l}$ with $\max \{r\}=n-1$, where $\mathbb{P}=\{1,2, \ldots\}$. Set $r^{\prime}=(r, k), 1 \leq k \leq n-1$. Then for any $w \in \mathfrak{S}_{n}$ and $w \preceq r^{\prime}$, we have

1. If $w \npreceq r$, then $\alpha_{r^{\prime}}(w)=\alpha_{r}\left(w s_{k}\right) \cdot \boldsymbol{k}$,

2. If $w \preceq r, w s_{k} \npreceq r$, then $\alpha_{r^{\prime}}(w)=\alpha_{r}(w)$,

3. If $w, w s_{k} \preceq r$, and $\ell\left(w s_{k}\right)=\ell(w)+1$, then $\alpha_{r^{\prime}}(w)=\alpha_{r}(w)+\alpha_{r}\left(w s_{k}\right) \cdot \boldsymbol{k} q$,

4. If $w, w s_{k} \preceq r$, and $\ell\left(w s_{k}\right)=\ell(w)-1$, then $\alpha_{r^{\prime}}(w)=\alpha_{r}(w) \cdot q^{k}+\alpha_{r}\left(w s_{k}\right) \cdot \boldsymbol{k}$.

Proof We have

$$
Q\left(r^{\prime}\right)=Q(r)\left(1+\boldsymbol{k} T_{k}\right)=\sum_{w \preceq r} \alpha_{r}(w) T_{w}+\sum_{u \preceq r} \alpha_{r}(u) T_{u} \cdot \boldsymbol{k} T_{k} .
$$


We will prove the desired result by applying (2), and comparing the coefficients of $T_{w}$ on both sides of (5).

1. If $w, w s_{k} \npreceq r$ then we have $\alpha_{r^{\prime}}(w)=0=\alpha_{r}\left(w s_{k}\right) \cdot \boldsymbol{k}$. If $w \npreceq r$ and $w s_{k} \preceq r$ then $T_{w}$ can only be obtained by $T_{w s_{k}} \cdot \boldsymbol{k} T_{k}$, so we have $\alpha_{r^{\prime}}(w)=\alpha_{r}\left(w s_{k}\right) \cdot \boldsymbol{k}$.

2. If $w \preceq r$ and $w s_{k} \npreceq r$, then there is no $u \preceq r$ such that $u s_{k}=w$. Hence $T_{w}$ can only be obtained by $T_{w} \cdot 1$, so we have $\alpha_{r^{\prime}}(w)=\alpha_{r}(w)$.

3. If $w, w s_{k} \preceq r$ and $\ell\left(w s_{k}\right)=\ell(w)+1$, then $T_{w} \cdot \boldsymbol{k} T_{k}=\boldsymbol{k} T_{w s_{k}}$, and there is $u=$ $w \cdot s_{k} \preceq r$ such that $T_{u} \cdot \boldsymbol{k} T_{k}=\boldsymbol{k}\left((q-1) T_{u}+q T_{u \cdot s_{k}}\right)=\boldsymbol{k}\left((q-1) T_{w s_{k}}+q T_{w}\right)$. Therefore we have $\alpha_{r^{\prime}}(w)=\alpha_{r}(w)+\alpha_{r}(u) \cdot \boldsymbol{k} q=\alpha_{r}(w)+\alpha_{r}\left(w s_{k}\right) \cdot \boldsymbol{k} q$.

4. If $w, w s_{k} \preceq r$ and $\ell\left(w s_{k}\right)=\ell(w)-1$, then $T_{w} \cdot \boldsymbol{k} T_{k}=\boldsymbol{k}\left((q-1) T_{w}+q T_{w s_{k}}\right)$, and there is $u=w \cdot s_{k} \preceq r$ such that $T_{u} \cdot \boldsymbol{k} T_{k}=\boldsymbol{k} T_{u \cdot s_{k}}=\boldsymbol{k} T_{w}$. Therefore we have

$$
\begin{aligned}
\alpha_{r^{\prime}}(w) & =\alpha_{r}(w)+\alpha_{r}(u) \cdot \boldsymbol{k}+\alpha_{r}(w) \cdot \boldsymbol{k}(q-1) \\
& =\alpha_{r}(w) \cdot q^{k}+\alpha_{r}\left(w s_{k}\right) \cdot \boldsymbol{k} .
\end{aligned}
$$

We also want to list the following result related to $\operatorname{inv}(w)$ and $\operatorname{inv}\left(w s_{k}\right)$, which is frequently used in the proof of Theorem 1 . The proof of this result is quite straightforward and is omitted here.

Lemma 2 For any permutation $w \in \mathfrak{S}_{n}$ and adjacent transposition $s_{k}, 1 \leq k \leq n-1$, we have the following properties of the statistic inv $w_{w}$.

1. If $\ell\left(w s_{k}\right)=\ell(w)-1$, then

$$
\begin{aligned}
& \operatorname{inv}_{w}(k)>\operatorname{inv}_{w}(k+1), \operatorname{inv}_{w s_{k}}(k)=\operatorname{inv}_{w}(k+1), \\
& \text { and } \operatorname{inv}_{w s_{k}}(k+1)=\operatorname{inv}_{w}(k)-1 .
\end{aligned}
$$

2. If $\ell\left(w s_{k}\right)=\ell(w)+1$ then

$$
\begin{aligned}
& \operatorname{inv}_{w}(k) \leq \operatorname{inv}_{w}(k+1), \operatorname{inv}_{w s_{k}}(k)=\operatorname{inv}_{w}(k+1)+1 \\
& \quad \text { and } \operatorname{inv}_{w s_{k}}(k+1)=\operatorname{inv}_{w}(k) .
\end{aligned}
$$

We need one more lemma before we prove the main theorem.

Lemma 3 Let $r$ be a tight sequence, and $w$ be a permutation such that $w \preceq r$, then $\operatorname{inv}_{w}(i) \leq a_{r}(i)$ for any $i \geq 1$.

Proof We will prove this result by induction on the length of $r$. The case for $r=(1)$ is trivial. Suppose the result is true for some tight sequence $r$, and $r^{\prime}=(r, k)$ is also a tight sequence. For any permutation $w \preceq r^{\prime}$, if $w \preceq r$, we have

$$
\operatorname{inv}_{w}(i) \leq a_{r}(i) \leq a_{r^{\prime}}(i), \quad \forall i \geq 1
$$

If $w \npreceq r$, we will first prove that in this case $a_{r}(k)=a_{r}(k+1)$. If $a_{r}(k)=0$, it is obvious that $a_{r}(k)=a_{r}(k+1)=0$. If $a_{r}(k)>0$, then since $w \preceq r^{\prime}$ but $w \npreceq r$, 
there must be a $k+1$ to the right of the rightmost $k$ in $r$. Suppose this $k+1$ occurs at the $j$ th position in $r$. We have that $\left(r_{1}, r_{2}, \ldots, r_{j-1}, r_{j}\right)=\left(r_{1}, r_{2}, \ldots, r_{j-1}\right.$, $k+1)$ is a tight sequence, with some permutation $w^{\prime} \preceq\left(r_{1}, r_{2}, \ldots, r_{j-1}, r_{j}\right)$ but $w^{\prime} \npreceq\left(r_{1}, r_{2}, \ldots, r_{j-1}\right)$. Thus we have $a_{\left(r_{1}, \ldots, r_{j-1}\right)}(k+1)=a_{\left(r_{1}, \ldots, r_{j-1}\right)}(k)-1$. Therefore $a_{r}(k)=a_{\left(r_{1}, \ldots, r_{j-1}\right)}(k)=a_{\left(r_{1}, \ldots, r_{j-1}\right)}(k+1)+1=a_{r}(k+1)$.

Moreover, since $w \npreceq r$, there exists a permutation $u \npreceq r$ such that $w=u s_{k}$ and $l(w)=l(u)+1$. It is obvious that for $i \neq k, k+1$, we have $\operatorname{inv}_{w}(i)=\operatorname{inv}_{u}(i) \leq$ $a_{r}(i)=a_{r^{\prime}}(i)$. Moreover, from Lemma 2 we know that

$$
\operatorname{inv}_{w}(k)=\operatorname{inv}_{u}(k+1)+1 \leq a_{r}(k+1)+1=a_{r}(k)+1=a_{r^{\prime}}(k),
$$

and

$$
\operatorname{inv}_{w}(k+1)=\operatorname{inv}_{u}(k) \leq a_{r}(k)=a_{r}(k+1)=a_{r^{\prime}}(k+1) .
$$

Hence the proof is complete.

Now we are ready to prove the main theorem.

Proof of Theorem 1 The proof is by induction on $l$, the length of the sequence $r$. It is trivial to check that (3) holds for $r=(1)$. Suppose that (3) holds for some tight sequence $r$, and $r^{\prime}=(r, k)$ is also a tight sequence. We want to prove that (3) also holds for $r^{\prime}$. The case when $k>\max \{r\}$ is trivial, so from now on we will assume that $\max \{r\}=\max \left\{r^{\prime}\right\}=n-1$.

For any $i \neq k, k+1(1 \leq i \leq n-1)$, we have $a_{r^{\prime}}(i)=a_{r}(i)$, and $\operatorname{inv}_{w}(i)=$ $\operatorname{inv}_{w s_{k}}(i)$. Therefore

$$
\begin{aligned}
\max \left\{a_{r^{\prime}}(i-1)-1, \operatorname{inv}_{w}(i)\right\} & =\max \left\{a_{r}(i-1)-1, \operatorname{inv}_{w}(i)\right\} \\
& =\max \left\{a_{r}(i-1)-1, \operatorname{inv}_{w s_{k}}(i)\right\}
\end{aligned}
$$

holds for any $i \neq k, k+1(2 \leq i \leq n-1)$. Hence we only need to concentrate on the values of $\max \left\{a_{r^{\prime}}(i-1)-1, \operatorname{inv}_{w}(i)\right\}$ for $i=k, k+1$. (When $k=1$, we only consider $\max \left\{a_{r^{\prime}}(1)-1, \operatorname{inv}_{w}(2)\right\}$.)

Next we will prove that $\alpha_{r^{\prime}}(w)=\prod_{i=2}^{n-1} i^{\max \left\{a_{r^{\prime}}(i-1)-1, \operatorname{inv}_{w}(i)\right\}}$ for any $w \npreceq r^{\prime}$ according to the four cases in Lemma 1 , and we will frequently use Lemma 2.

1. Let $w \npreceq r$. In this case $\operatorname{inv}_{w}(k)=a_{r}(k)+1=a_{r^{\prime}}(k) \leq n-k$. Since $r, r^{\prime}$ are both tight sequences we have $a_{r^{\prime}}(k-1)=a_{r}(k-1)=a_{r}(k)+1$. Moreover, since $\operatorname{inv}_{w s_{k}}(k)=\operatorname{inv}_{w}(k+1)<\operatorname{inv}_{w}(k)=a_{r}(k)+1$, we have

$$
\max \left\{a_{r^{\prime}}(k-1)-1, \operatorname{inv}_{w}(k)\right\}=a_{r}(k)+1=\max \left\{a_{r}(k-1)-1, \operatorname{inv}_{w s_{k}}(k)\right\}+1 .
$$

Since $\operatorname{inv}_{w s_{k}}(k+1)=\operatorname{inv}_{w}(k)-1=a_{r}(k)$ and $a_{r^{\prime}}(k)=a_{r}(k)+1$, we have

$$
\max \left\{a_{r^{\prime}}(k)-1, \operatorname{inv}_{w}(k+1)\right\}=\max \left\{a_{r}(k)-1, \operatorname{inv}_{w s_{k}}(k+1)\right\}=a_{r}(k) .
$$


Hence we conclude that

$$
\begin{aligned}
\alpha_{r^{\prime}}(w)=\alpha_{r}\left(w s_{k}\right) \cdot \boldsymbol{k} & =\prod_{i=2}^{n-1} \boldsymbol{i}^{\max \left\{a_{r}(i-1)-1, \operatorname{inv}_{w_{k}}(i)\right\}} \cdot \boldsymbol{k} \\
& =\prod_{i=2}^{n-1} \boldsymbol{i}^{\max \left\{a_{r^{\prime}}(i-1)-1, \operatorname{inv}_{w}(i)\right\}} .
\end{aligned}
$$

2. Let $w \preceq r$ and $w s_{k} \npreceq r$. In this case we have $\ell\left(w s_{k}\right)=\ell(w)+1$ and $\operatorname{inv}_{w}(k)=$ $a_{r}(k)$.

Since $\operatorname{inv}_{w}(k+1) \geq \operatorname{inv}_{w}(k)=a_{r}(k)$ and $a_{r^{\prime}}(k)=a_{r}(k)+1$, we have

$$
\max \left\{a_{r}(k)-1, \operatorname{inv}_{w}(k+1)\right\}=\max \left\{a_{r^{\prime}}(k)-1, \operatorname{inv}_{w}(k+1)\right\} .
$$

It follows that $\alpha_{r^{\prime}}(w)=\alpha_{r}(w)=\prod_{i=2}^{n-1} i^{\max \left\{a_{r^{\prime}}(i-1)-1, \operatorname{inv}_{w}(i)\right\}}$.

3. Let $w, w s_{k} \preceq r$ and $\ell\left(w s_{k}\right)=\ell(w)+1$. Since $\operatorname{inv}_{w}(k)<a_{r}(k), \operatorname{inv}_{w s_{k}}(k) \leq a_{r}(k)$ and $a_{r}(k-1)-1 \geq a_{r}(k)$, we have

$$
\max \left\{a_{r}(k-1)-1, \operatorname{inv}_{w}(k)\right\}=\max \left\{a_{r}(k-1)-1, \operatorname{inv}_{w s_{k}}(k)\right\}=a_{r}(k-1)-1 .
$$

Since $\operatorname{inv}_{w}(k+1)=\operatorname{inv}_{w s_{k}}(k)-1 \leq a_{r}(k)-1$, and $\operatorname{inv}_{w s_{k}}(k+1)=\operatorname{inv}_{w}(k)<$ $a_{r}(k)$, we have

$$
\max \left\{a_{r}(k)-1, \operatorname{inv}_{w}(k+1)\right\}=\max \left\{a_{r}(k)-1, \operatorname{inv}_{w s_{k}}(k+1)\right\}=a_{r}(k)-1 .
$$

Hence $\alpha_{r}(w)=\alpha_{r}\left(w s_{k}\right)$. Therefore we have

$$
\begin{aligned}
\alpha_{r^{\prime}}(w)=\alpha_{r}(w)+\alpha_{r}\left(w s_{k}\right) \cdot \boldsymbol{k} q & =\alpha_{r}(w)(\boldsymbol{k}+\mathbf{1}) \\
& =\prod_{i=2}^{n-1} \boldsymbol{i}^{\max \left\{a_{r}(i-1)-1, \operatorname{inv}_{w}(i)\right\}} \cdot(\boldsymbol{k}+\mathbf{1}) .
\end{aligned}
$$

Moreover, since $\max \left\{a_{r^{\prime}}(k)-1, \operatorname{inv}_{w}(k+1)\right\}=a_{r}(k)=\max \left\{a_{r}(k)-1\right.$, $\left.\operatorname{inv}_{w}(k+1)\right\}+1$, we have $\alpha_{r^{\prime}}(w)=\prod_{i=2}^{n-1} i^{\max \left\{a_{r^{\prime}}(i-1)-1, \operatorname{inv}_{w}(i)\right\}}$.

4. Let $w, w s_{k} \preceq r$ and $\ell\left(w s_{k}\right)=\ell(w)-1$. In this case $\operatorname{inv}_{w}(k) \leq a_{r}(k)$.

Since $\operatorname{inv}_{w s_{k}}(k)=\operatorname{inv}_{w}(k+1)<\operatorname{inv}_{w}(k) \leq a_{r}(k)$ and $a_{r}(k-1)-1 \geq a_{r}(k)$, we have

$$
\max \left\{a_{r}(k-1)-1, \operatorname{inv}_{w}(k)\right\}=\max \left\{a_{r}(k-1)-1, \operatorname{inv}_{w s_{k}}(k)\right\}=a_{r}(k-1)-1 .
$$

Since $\operatorname{inv}_{w s_{k}}(k+1)=\operatorname{inv}_{w}(k)-1$, we have

$$
\max \left\{a_{r}(k)-1, \operatorname{inv}_{w}(k+1)\right\}=\max \left\{a_{r}(k)-1, \operatorname{inv}_{w s_{k}}(k+1)\right\}=a_{r}(k)-1 .
$$


Hence $\alpha_{r}(w)=\alpha_{r}\left(w s_{k}\right)$. Therefore we have

$$
\begin{aligned}
\alpha_{r^{\prime}}(w)=\alpha_{r}(w) \cdot q^{k}+\alpha_{r}\left(w s_{k}\right) \cdot \boldsymbol{k} & =\alpha_{r}(w) \cdot(\boldsymbol{k}+\mathbf{1}) \\
& =\prod_{i=2}^{n-1} \boldsymbol{i}^{\max \left\{a_{r}(i-1)-1, \operatorname{inv}_{w}(i)\right\}} \cdot(\boldsymbol{k}+\mathbf{1}) .
\end{aligned}
$$

Moreover, since $\max \left\{a_{r^{\prime}}(k)-1, \operatorname{inv}_{w}(k+1)\right\}=a_{r}(k)=\max \left\{a_{r}(k)-1\right.$, $\left.\operatorname{inv}_{w}(k+1)\right\}+1$, we have $\alpha_{r^{\prime}}(w)=\prod_{i=2}^{n-1} i^{\max \left\{a_{r^{\prime}}(i-1)-1, \operatorname{inv}_{w}(i)\right\}}$.

Hence the proof is complete.

We can use Theorem 1 and its proof to compute $\alpha_{r}(w)$ for certain sequences $r$ that are not tight sequences.

Corollary 1 Let $r$ be a sequence of positive integers, and $\max \{r\}=n-1$. If $r$ has the prefix $\rho_{n}=(1,2,1,3,2,1 \ldots, n, n-1, \ldots, 1)$, then we have

$$
\alpha_{r}(w)=\prod_{i=2}^{n-1} i^{\max \left\{a_{r}(i-1)-1, \operatorname{inv}_{w}(i)\right\}} .
$$

Proof We will prove equation (6) by induction on the length of $r$. Since $\rho_{n}$ is a tight sequence, from Theorem 1 we know that the result holds for $r=\rho_{n}$. Next assume the result for $r$ and let $r^{\prime}=(r, k)$ with $1 \leq k \leq n-1$. We do an induction similar to what we did in the proof of Theorem 1. Since $r$ has the prefix $\rho_{n}$, it follows that for any $w \in \mathfrak{S}_{n}, w, w s_{k} \preceq r$. Therefore only cases 3 and 4 will occur. Moreover, since $a_{r}(k-1) \geq n-(k-1), a_{r}(k) \geq n-k$ and $a_{r^{\prime}}(k)=a_{r}(k)+1$, we have

$$
\begin{aligned}
& \max \left\{a_{r}(k-1)-1, \operatorname{inv}_{w}(k)\right\}=\max \left\{a_{r}(k-1)-1, \operatorname{inv}_{w s_{k}}(k)\right\}=a_{r}(k-1)-1, \\
& \max \left\{a_{r}(k)-1, \operatorname{inv}_{w}(k+1)\right\}=\max \left\{a_{r}(k)-1, \operatorname{inv}_{w s_{k}}(k+1)\right\}=a_{r}(k)-1,
\end{aligned}
$$

and

$$
\max \left\{a_{r^{\prime}}(k)-1, \operatorname{inv}_{w}(k+1)\right\}=a_{r}(k)=\max \left\{a_{r}(k)-1, \operatorname{inv}_{w}(k+1)\right\}+1 .
$$

Hence for both case 3 and 4 we have $\alpha_{r^{\prime}}(w)=\prod_{i=2}^{n-1} i^{\max \left\{a_{r^{\prime}}(i-1)-1, \operatorname{inv}_{w}(i)\right\}}$.

Note that $r$ is a reduced decomposition of $w \in \mathfrak{S}_{n}$ if and only if the reverse of $r$ is a reduced decomposition of $w^{-1}$. Thus we have the following result.

Corollary 2 Let $r$ be a sequence of positive integers, and $\max \{r\}=n-1$. If

1. $r$ is the reverse of a tight sequence, or

2. $r$ has suffix $\rho_{n}=(1,2,1,3,2,1 \ldots, n, n-1, \ldots, 1)$,

then for any $w \in \mathfrak{S}_{n}$ and $w \preceq r$, we have

$$
\alpha_{r}(w)=\prod_{i=2}^{n-1} i^{\max \left\{a_{r}(i-1)-1, \operatorname{inv}_{w^{-1}}(i)\right\}} .
$$


Note. If a sequence $r^{\prime}$ is obtained from $r$ by transposing two adjacent terms that differ by at least 2, then $Q(r)=Q\left(r^{\prime}\right)$, so $\alpha_{w}(r)=\alpha_{w}\left(r^{\prime}\right)$. Thus our results extend to sequences that can be obtained from those of Theorem 1, Corollary 1, and Corollary 2 by applying such "commuting transpositions" to $r$.

\section{A connection with random walks on $\mathfrak{S}_{n}$}

There is a huge literature on random walks on $\mathfrak{S}_{n}$, e.g., [3]. Our results can be interpreted in this context. First consider the case $q=1$. In this case the Hecke algebra $\mathcal{H}_{n}(q)$ reduces to the group algebra $\mathbb{R} \mathfrak{S}_{n}$ of $\mathfrak{S}_{n}$, and the generator $T_{i}$ becomes the adjacent transposition $s_{i}$. Thus

$$
Q(r)_{q=1}=\left(1+r_{1} s_{r_{1}}\right)\left(1+r_{2} s_{r_{2}}\right) \cdots\left(1+r_{l} s_{r_{l}}\right) .
$$

We normalize this expression by dividing each factor $1+r_{i} s_{r_{i}}$ by $1+r_{i}$. Write

$$
D_{j}=\left(1+j s_{j}\right) /(1+j),
$$

and set

$$
\widetilde{Q}(r)=D_{r_{1}} D_{r_{2}} \cdots D_{r_{l}} .
$$

If $P$ is a probability distribution on $\mathfrak{S}_{n}$, then let $\sigma_{P}=\sum_{w \in \mathfrak{S}_{n}} P(w) w \in \mathbb{R} \mathfrak{S}_{n}$. If $P^{\prime}$ is another probability distribution on $\mathfrak{S}_{n}$, then $\sigma_{P} \sigma_{P^{\prime}}=\sigma_{P * P^{\prime}}$ for some probability distribution $P * P^{\prime}$, the convolution of $P$ and $P^{\prime}$. It follows that $\widetilde{Q}(r)=\sigma_{P_{r}}$ for some probability distribution $P_{r}$ on $\mathfrak{S}_{n}$. Theorem 1 gives (after setting $q=1$ and normalizing) an explicit formula for the distribution $P_{r}$, i.e., the values $P_{r}(w)$ for all $w \in \mathfrak{S}_{n}$. Note in particular that if $r$ is the standard tight sequence $\rho_{n}=$ $(1,2,1,3,2,1,4,3,2,1, \ldots, n-1, n-2, \ldots, 1)$, then from equation (4) we get

$$
\widetilde{Q}\left(\rho_{n}\right)=\frac{1}{n !} \sum_{w \in \mathfrak{S}_{n}} w=\sigma_{U},
$$

where $U$ is the uniform distribution on $\mathfrak{S}_{n}$. (We have been informed by Alexander Molev that an equivalent result was given by Jucys [6] in 1966. We have also been informed by Persi Diaconis that this result, and similar results for some other groups, were known by him and Colin Mallows twenty years ago.) It is not hard to see directly why we obtain the uniform distribution. Namely, start with any permutation $w=$ $w_{1} \cdots w_{n} \in \mathfrak{S}_{n}$. Do nothing with probability $1 / 2$ or apply $s_{1}$ (i.e., interchange $w_{1}$ and $w_{2}$ ) with probability $1 / 2$, obtaining $y_{1} y_{2} w_{3} \cdots w_{n}$. Thus $y_{2}$ is equally likely to be $w_{1}$ or $w_{2}$. Now either do nothing with probability $1 / 3$ or apply $s_{2}$ with probability $2 / 3$, obtaining $y_{1} z_{2} z_{3} w_{4} \cdots w_{n}$. Then $z_{3}$ is equally likely to be $w_{1}, w_{2}$ or $w_{3}$. Continue in this way, applying $s_{3}, \ldots, s_{n-1}$ at each step or doing nothing, with probability $1 /(i+1)$ of doing nothing at the $i$ th step, obtaining $d_{1} \cdots d_{n}$. Then $d_{n}$ is equally likely to be any of $1,2, \ldots, n$. Now apply $s_{1}, s_{2}, \ldots, s_{n-2}$ or do nothing as before, obtaining $e_{1} \cdots e_{n}$. The last element $e_{n}$ has never switched, so $e_{n}=d_{n}$, and now $e_{n-1}$ is equally likely to be any element of $\{1,2, \ldots, n\}-\left\{d_{n}\right\}$. Continue as before with 
$s_{1}, \ldots, s_{n-3}$, then $s_{1}, \ldots, s_{n-4}$, etc., ending in $s_{1}, s_{2}, s_{1}$, at which point we obtain a uniformly distributed random permutation.

Now consider the situation for $\mathcal{H}_{n}(q)$. If $P$ is a probability distribution on $\mathfrak{S}_{n}$ then write $\tau_{P}=\sum_{w \in \mathfrak{S}_{n}} P(w) T_{w} \in \mathcal{H}_{n}(q)$. If $P^{\prime}$ is another probability distribution on $\mathfrak{S}_{n}$, then in general it is not true that $\tau_{P} \tau_{P}^{\prime}=\tau_{R}$ for some probability distribution $R$. A probabilistic interpretation of Theorem 1 requires the use of a Markov chain. Let $0<q<1$. Note that from equation (2) we have

$$
T_{w}\left(1+\boldsymbol{k} T_{k}\right)= \begin{cases}T_{w}+\boldsymbol{k} T_{w s_{k}}, & \ell\left(w s_{k}\right)=\ell(w)+1, \\ q^{k} T_{w}+q \boldsymbol{k} T_{w s_{k}}, & \ell\left(w s_{k}\right)=\ell(w)-1 .\end{cases}
$$

Divide each side by $1+\boldsymbol{k}$. Let $w=w_{1} \cdots w_{n}$. We can then interpret multiplication of $T_{w}$ by $\left(1+\boldsymbol{k} T_{w}\right) /(1+\boldsymbol{k})$ as follows. If $w_{k}<w_{k+1}$ then transpose $w_{k}$ and $w_{k+1}$ with probability $\boldsymbol{k} /(1+\boldsymbol{k})$, or do nothing with probability $1 /(1+\boldsymbol{k})$. If $w_{k}>w_{k+1}$, then transpose $w_{k}$ and $w_{k+1}$ with probability $q \boldsymbol{k} /(1+\boldsymbol{k})$, or do nothing with probability $q^{k} /(1+\boldsymbol{k})$. Since

$$
\frac{q \boldsymbol{k}}{1+\boldsymbol{k}}+\frac{q^{k}}{1+\boldsymbol{k}}<1
$$

we have a "leftover" probability of $\left(1-\left(q \boldsymbol{k}+q^{k}\right) /(1+\boldsymbol{k})\right)$. In this case the process has failed and we should start it all over. Let us call this procedure a $k$-step.

If $r=\left(r_{1}, \ldots, r_{l}\right)$ is a tight sequence, then begin with the identity permutation and apply an $r_{1}$-step, $r_{2}$-step, etc. If we need to start over, then we again begin with the identity permutation and apply an $r_{1}$-step, $r_{2}$-step, etc. Eventually (with probability 1) we will apply $r_{i}$-steps for all $1 \leq i \leq l$, ending with a random permutation $v$. In this case, Theorem 1 tells us the distribution of $v$, namely, the probability of $v$ is

$$
P(v)=\frac{\alpha_{r}(v)}{\prod_{i=1}^{l}\left(1+r_{i}\right)}
$$

In particular, if $r$ is the standard tight sequence $\rho_{n}$, then $v$ is uniformly distributed.

Example 2 Start with the permutation 123 and $r=\rho_{3}=(1,2,1)$. Let us calculate by "brute force" the probability $P=P(123)$ that $v=123$. There are three ways to achieve $v=123$.

(a) Apply a 1-step, a 2-step, and a 1-step, doing nothing each time. This has probability $(1 / 2)(1 /(2+q))(1 / 2)=1 / 4(2+q)$.

(b) Apply a 1-step and switch. Apply a 2-step and do nothing. Apply a 1-step and switch. This has probability $q / 4(2+q)$.

(c) Apply a 1-step and switch. Apply a 2-step and do nothing. Try to apply a 1-step but go back to the beginning, after which we continue the process until ending up with 123 . This has probability

$$
\frac{1}{2} \frac{1}{2+q}(1-q) P=\frac{P(1-q)}{2(2+q)} .
$$


Hence

$$
P=\frac{1}{4(2+q)}+\frac{q}{4(2+q)}+\frac{P(1-q)}{2(2+q)} .
$$

Solving for $P$ gives (somewhat miraculously!) $P=1 / 6$. Similarly for all other $w \in \mathfrak{S}_{3}$ we get $P(w)=1 / 6$.

NOTE. A probabilistic interpretation of certain Hecke algebra products different from ours appears in a paper by Diaconis and Ram [4].

\section{References}

1. Cherednik, I.V.: Special bases of irreducible representations of a degenerate affine Hecke algebra. Funct. Analysis Appl. 20, 76-79 (1986). Translated from Funktsional. Anal. i Prilozhen. 20, 87-88 (1986)

2. Cherednik, I.V.: A new interpretation of Gel'fand Tzetlin bases. Duke Math. J. 54, 563-577 (1987)

3. Diaconis, P.: Group Representations in Probability and Statistics. Lecture Notes-Monograph Series, vol. 11. Institute of Mathematical Statistics, Hayward (1988)

4. Diaconis, P., Ram, A.: Analysis of systematic scan Metropolis algorithms using Iwahori-Hecke algebra techniques. Michigan Math. J. 48, 157-190 (2000)

5. Humphreys, J.E.: Reflection Groups and Coxeter Groups. Cambridge Studies in Advanced Mathematics, vol. 29. Cambridge University Press, Cambridge (1990)

6. Jucys, A.: On the Young operators of symmetric groups. Litovsk. Fiz. Sb. 6, 163-180 (1966) 